This item was submitted to Loughborough's Research Repository by the author.

Items in Figshare are protected by copyright, with all rights reserved, unless otherwise indicated.

\title{
Throughput maximization for full-duplex energy harvesting MIMO
} \section{communications}

PLEASE CITE THE PUBLISHED VERSION

http://dx.doi.org/10.1109/SPAWC.2016.7536806

PUBLISHER

(C) IEEE

VERSION

AM (Accepted Manuscript)

LICENCE

CC BY-NC-ND 4.0

\section{REPOSITORY RECORD}

Chalise, Batu K., Himal A. Suraweera, and Gan Zheng. 2019. "Throughput Maximization for Full-duplex Energy Harvesting MIMO Communications”. figshare. https://hdl.handle.net/2134/23286. 


\title{
Throughput Maximization for Full-Duplex Energy Harvesting MIMO Communications
}

\author{
Batu K. Chalise*, Himal A. Suraweera ${ }^{\dagger}$, and Gan Zheng ${ }^{\ddagger}$ \\ ${ }^{*}$ Department of Electrical Engineering and Computer Science, Cleveland State University, USA \\ ${ }^{\dagger}$ Department of Electrical and Electronic Engineering, University of Peradeniya, Sri Lanka \\ $\ddagger$ Wolfson School of Mechanical, Electrical and Manufacturing Engineering, Loughborough University, UK \\ Email: batu.k.chalise@ieee.org, himal@ee.pdn.ac.lk, g.zheng@lboro.ac.uk
}

\begin{abstract}
This paper proposes methods for optimizing bidirectional information rates between a base station (BS) and a wirelessly powered mobile station (MS). In the first phase, the MS harvests energy using signals transmitted by the BS, whereas in the second phase both the BS and MS communicate to each other in a full-duplex mode. The BS-beamformer and the time-splitting parameter (TSP) of energy harvesting scheme are jointly optimized to obtain the BS-MS rate region. The joint optimization is non-convex, however a computationally efficient optimum technique based upon semidefinite relaxation and linesearch is proposed to solve the problem. Moreover, a suboptimum approach based upon the zero-forcing (ZF) beamformer constraint is also proposed. In this case, a closed-form solution of TSP is obtained. Simulation results demonstrate the advantage of the optimum method over the suboptimum method, especially for smaller values of BS transmit power and number of transmit antennas at the $\mathrm{BS}$.
\end{abstract}

\section{INTRODUCTION}

Currently most bi-directional wireless systems have been developed assuming half-duplex (HD) operation [1]. As a way of improving the spectral efficiency of contemporary HD systems, full-duplex (FD) communications can be used. Although the concept of FD is not new, it has been considered as impossible to date due to large loopback interference (LI) [2], [3]. However, FD is now becoming feasible thanks to promising analog and digital LI cancellation techniques that can achieve high transmit-receive isolation [4]-[6].

In addition to spectral efficiency, the issue of energy efficiency has gained wide research attention for the design of future wireless networks. For example, energy constraints impose an upper limit on transmit power and associated signal processing in wireless devices. To this end, a new communication paradigm that can power devices by utilizing wireless energy transfer (WET) has emerged [7]-[9].

Some existing works in the literature have investigated point-to-point (P2P) bi-directional FD wireless systems. These include papers that have focused on informationcommunication theoretic metrics such as achievable sum rates and the symbol error probability. In [3], achievable upper and lower sum-rate bounds of multiple antenna bi-directional communication that use pilot-aided channel estimates for transmit/receive beamforming and interference cancellation were derived. The beamforming performance of bi-directional multiple-input multiple-output (MIMO) transmission with spatial LI mitigation was investigated in [10]. The capacity of a bi-directional MIMO system with spatial fading correlation was presented in [11]. The maximization of the asymptotic ergodic mutual information for a MIMO bi-directional communication system with imperfect channel state information (CSI) assumptions was the focus of [12].

Motivated by the advantages of FD and WET, some recent works have also investigated the performance of wirelesspowered P2P bi-directional FD [13]-[15]. In [13], considering a hybrid FD access-point (AP) that broadcasts wireless energy to a set of downlink users while receiving information from a set of uplink users, a solution to an optimal resource allocation problem was presented. In [14], hardware implementation of a wireless system that transmits data and power in the same frequency was presented. More recently, in [15], a weighted sum transmit power optimization problem for a bi-directional P2P FD system with WET was formulated and solved. However, it neglected an important aspect of FD operation, namely, perfect LI cancellation was assumed at each terminal.

Inspired by wireless-powered FD communications, in this paper, we consider bi-directional communication between an $N$-antenna base station (BS) and an mobile station (MS) with two antennas. In our "harvest-then-transmit" system, the BS first transmits energy to the MS which will be used by the MS for subsequent uplink transmission. At the end of energy transfer phase, both BS and MS simultaneously transfer information in the uplink and downlink. The boundary of the BS-MS information rate region is characterized, which describes the trade-offs between BS and MS information rates. We propose optimum and suboptimum methods for jointly optimizing the beamformer at the BS and the time-splitting parameter (TSP) that divides a given time-slot into energy harvesting and data transmission phases. A computationally efficient optimum method based upon semidefinite relaxation (SDR) and line-search is proposed, whereas the suboptimum method uses the zero-forcing (ZF) criterion for designing the beamformer. In the latter case, a closed-form solution of TSP is also derived. Simulation results demonstrate that the proposed optimum method outperforms the suboptimum method, especially when BS transmit power and number of transmit antennas at the BS assume low values.

The rest of the paper is organized as follows. The system model and problem formulation are presented in Section II. The proposed optimum and suboptimum methods are solved 
in Section III, whereas in Section IV simulation results are presented. Finally, the conclusions are drawn in Section V.

\section{System Model AND PROBlem Formulation}

We consider bidirectional communications between an $N$ antenna BS and a MS with two antennas [15]. Specifically, the BS has $N_{t}$ transmit antennas and $\left(N-N_{t}\right)$ receive antennas. This number, $N_{t}$, together with the associated transmit/receive chosen antennas could be optimized, but we keep them fixed. At the MS side, one antenna is for transmission and the other is for reception. Since the MS is usually power limited and the uplink rate is the bottleneck, we consider a case where the BS first transmits energy to the MS, which will be used by the MS for the subsequent uplink transmission.

The communication takes place in two phases with duration $\alpha$ and $(1-\alpha)$, respectively. In phase I, the BS transmits energy to the MS. Suppose the transmit power of BS in this phase is $P$, then the received energy is

$$
E=\alpha P \lambda\left(\mathbf{H}_{B M} \mathbf{H}_{B M}^{H}\right),
$$

where the channel between the BS and the MS is denoted as $\mathbf{H}_{B M}$ and $\lambda(\cdot)$ returns the maximum eigenvalue of a matrix. In phase II, the BS and the MS communicate to each other using FD operation. The MS's transmit power can be written as

$$
p_{m}=\frac{\alpha \eta P \lambda\left(\mathbf{H}_{B M} \mathbf{H}_{B M}^{H}\right)}{1-\alpha},
$$

where $\eta$ is the conversion efficiency of wireless energy transfer. Assume that the BS's transmit power is no more than $P$ (not necessarily $P$ ) in this phase. Let the $1 \times N_{t} B S \rightarrow M S$ channel be $\mathbf{h}_{B}^{H}$ and the $\left(N-N_{t}\right) \times 1 M S \rightarrow B S$ channel is $\mathbf{h}_{M}$. The LI channels are $\mathbf{H}_{B} \in \mathbb{C}^{\left(N-N_{t}\right) \times N_{t}}$ and $h_{M}$ at the $\mathrm{BS}$ and MS, respectively. The respective transmit and receive beamformers at the BS are $\mathbf{w}_{B}$ and $\mathbf{r}_{B}$.

\section{A. Signal Model}

Received signals at the BS and the MS, are:

$$
\begin{array}{r}
y_{B}=\mathbf{r}_{B}^{H}\left(\sqrt{p_{m}} \mathbf{h}_{M} s_{M}+\mathbf{H}_{B} \mathbf{w}_{B} s_{B}+n_{B}\right), \\
y_{M}=\mathbf{h}_{B}^{H} \mathbf{w}_{B} s_{B}+\sqrt{p_{m}} h_{M} s_{M}+n_{M} .
\end{array}
$$

Then achievable rates (using the minimum mean-square error receiver at the $\mathrm{BS}$ ) are

$$
\begin{aligned}
r_{B} & =(1-\alpha) \log \left(1+\frac{p_{m}\left|\mathbf{r}_{B}^{H} \mathbf{h}_{M}\right|^{2}}{1+\left|\mathbf{r}_{B}^{H} \mathbf{H}_{B} \mathbf{w}_{B}\right|^{2}}\right) \\
& =(1-\alpha) \log \left(1+p_{m} \mathbf{h}_{M}^{H}\left(\mathbf{I}+\mathbf{H}_{B} \mathbf{w}_{B} \mathbf{w}_{B}^{H} \mathbf{H}_{B}^{H}\right)^{-1} \mathbf{h}_{M}\right) \\
& =(1-\alpha) \log \left(1+p_{m}\left(\left\|\mathbf{h}_{M}\right\|^{2}-\frac{\left|\mathbf{h}_{M}^{H} \mathbf{H}_{B} \mathbf{w}_{B}\right|^{2}}{1+\left\|\mathbf{H}_{B} \mathbf{w}_{B}\right\|^{2}}\right)\right)(4) \\
r_{M} & =(1-\alpha) \log \left(1+\frac{\left|\mathbf{h}_{B}^{H} \mathbf{w}_{B}\right|^{2}}{1+p_{m}\left|h_{m}\right|^{2}}\right) .
\end{aligned}
$$

\section{B. Problem Formulation}

We are interested to find the MS-BS rate region. This can be achieved by maximizing the MS rate while confirming that the BS-rate is equal to a certain value $R_{B}$. By solving this optimization problem for all $R_{B}$ where $R_{B} \in\left[0, R_{B}^{\max }\right]$ and $R_{B}^{\max }$ is the maximum value of BS rate, we obtain the MS$\mathrm{BS}$ rate region. Note that $R_{B}^{\max }$ is derived in closed-form in Appendix VI-A. As such, the optimization problem for a given $R_{B}$ is expressed as

$\max _{\left\|\mathbf{w}_{B}\right\|^{2} \leq P, 0 \leq \alpha \leq 1, p_{m}}(1-\alpha) \log \left(1+\frac{\left|\mathbf{h}_{B}^{H} \mathbf{w}_{B}\right|^{2}}{1+p_{m}\left|h_{m}\right|^{2}}\right)$ s.t.

$(1-\alpha) \log \left(1+p_{m}\left(\left\|\mathbf{h}_{M}\right\|^{2}-\frac{\left|\mathbf{h}_{M}^{H} \mathbf{H}_{B} \mathbf{w}_{B}\right|^{2}}{1+\left\|\mathbf{H}_{B} \mathbf{w}_{B}\right\|^{2}}\right)\right)=R_{B}$,

$p_{m}=\frac{\alpha \eta P \lambda\left(\mathbf{H}_{B M} \mathbf{H}_{B M}^{H}\right)}{1-\alpha}$.

The optimization problem (6) is a complicated nonconvex optimization w.r.t. $\mathbf{w}_{B}$ and $\alpha$. However, the problem can be solved efficiently by finding optimum $\mathbf{w}_{B}$ for a given $\alpha$ and vice-versa. Since $\alpha$ is scalar valued, the optimum solution can be ascertained by using one-dimensional search w.r.t. $\alpha$.

\section{PRoposed JoInt OptimizATION}

In this section, we propose optimum and suboptimum methods for solving the joint optimization of beamformer and the time-splitting parameter $(\alpha)$.

\section{A. Optimum Method}

In this method, the optimum $\mathbf{w}_{B}$ is found for a given $\alpha$. Since $\alpha$ is a scalar, the jointly optimal solution of $\mathbf{w}_{B}$ and $\alpha$ is obtained using one-dimensional search w.r.t. $\alpha$. The computational complexity of line search is minimized by exploiting the nature of the optimization problem (6).

1) Optimization of $\mathbf{w}_{B}$ : We first consider a problem to optimize $\mathbf{w}_{B}$ for a given $\alpha$. In this case, the optimization problem (6) is expressed as

$$
\begin{aligned}
& \max _{\left\|\mathbf{w}_{B}\right\|^{2} \leq P}(1-\alpha) \log \left(1+\frac{\left|\mathbf{h}_{B}^{H} \mathbf{w}_{B}\right|^{2}}{1+p_{m}\left|h_{m}\right|^{2}}\right) \text { s.t. } \\
& (1-\alpha) \log \left(1+p_{m}\left(\left\|\mathbf{h}_{M}\right\|^{2}-\frac{\left|\mathbf{h}_{M}^{H} \mathbf{H}_{B} \mathbf{w}_{B}\right|^{2}}{1+\left\|\mathbf{H}_{B} \mathbf{w}_{B}\right\|^{2}}\right)\right)=R_{B} .
\end{aligned}
$$

Since $\log (1+x)$ is a monotonically increasing function of $x$ and the denominator $1+p_{m}\left|h_{m}\right|^{2}$ of $x \triangleq \frac{\left|\mathbf{h}_{B}^{H} \mathbf{w}_{B}\right|^{2}}{1+p_{m}\left|h_{m}\right|^{2}}$ is independent of $\mathbf{w}_{B}$, (7) can be solved via

$$
\begin{aligned}
\max _{\left\|\mathbf{w}_{B}\right\|^{2} \leq P} & \left|\mathbf{h}_{B}^{H} \mathbf{w}_{B}\right|^{2} \\
\text { s.t. } & \frac{\left|\mathbf{h}_{M}^{H} \mathbf{H}_{B} \mathbf{w}_{B}\right|^{2}}{1+\left\|\mathbf{H}_{B} \mathbf{w}_{B}\right\|^{2}}=\Gamma_{B},
\end{aligned}
$$

where $\Gamma_{B} \triangleq\left\|\mathbf{h}_{M}\right\|^{2}-\frac{1}{p_{m}}\left[2^{\frac{R_{B}}{1-\alpha}}-1\right]$. It is clear that the objective function in (8) is maximized with $\left\|\mathbf{w}_{B}\right\|^{2}=P$. This optimization problem is nonconvex due to the fact that it is the maximization of a quadratic function with a quadratic equality constraint. Moreover, to the best of our knowledge, (8) does not admit a closed-form solution. However, it can be efficiently and optimally solved using semi-definite programming. For 
this purpose, define $\mathbf{V}_{B}=\mathbf{w}_{B} \mathbf{w}_{B}^{H}$ and relax the rank-one constraint $\operatorname{rank}\left(\mathbf{V}_{B}\right)=1$. The relaxed optimization is

$$
\begin{array}{cl}
\max _{\mathbf{V}_{B}} & f\left(\alpha, p_{m}\right)=\operatorname{tr}\left(\mathbf{V}_{B} \mathbf{h}_{B} \mathbf{h}_{B}^{H}\right) \\
\text { s.t. } & \operatorname{tr}\left(\mathbf{V}_{B} \mathbf{H}_{B}^{H} \mathbf{h}_{M} \mathbf{h}_{M}^{H} \mathbf{H}_{B}\right)=\Gamma_{B}\left(1+\operatorname{tr}\left(\mathbf{V}_{B} \mathbf{H}_{B}^{H} \mathbf{H}_{B}\right)\right), \\
& \operatorname{tr}\left(\mathbf{V}_{B}\right)=P, \mathbf{V}_{B} \succeq 0 .
\end{array}
$$

The optimization problem (9) is a standard SDR problem with only two equality constraints. Therefore, according to ShapiroBarvinok-Pataki (SBP) rank reduction theorem [16], there exists a rank-one optimum solution of $\mathbf{V}_{B}$ for this relaxed optimization problem. Let $\mathbf{V}_{B}^{*}$ be the optimum solution of (9). Since $\mathbf{V}_{B}^{*}$ is rank-one matrix, the optimum solution $\mathbf{w}_{B}^{*}$ is obtained $\mathbf{w}_{B}^{*}=\sqrt{P} \tilde{\mathbf{u}} \tilde{\mathbf{u}}^{H}$, where $\tilde{\mathbf{u}}$ is the eigenvector corresponding to non-zero eigenvalue of $\mathbf{V}_{B}^{*}$.

2) Optimization of $\mathbf{w}_{B}$ and $\alpha$ : In order to jointly optimize $\mathbf{w}_{B}$ and $\alpha$, we solve the SDR problem (9) using onedimensional (or line search) search over $\alpha$ where $0 \leq \alpha \leq 1$. However, this line search can be limited to a small segment, and therefore, the number of required SDR optimizations can be significantly minimized. To illustrate this, let the objective function in (7), for a given $\mathbf{w}_{B}^{*}$, be defined as

$$
f(\alpha)=(1-\alpha) \log _{2}\left(1+\frac{\beta}{c+\frac{\alpha b}{1-\alpha}}\right)
$$

where

$$
\beta=\frac{\left|\mathbf{h}_{B}^{H} \mathbf{w}_{B}^{*}\right|^{2}}{\left|h_{m}\right|^{2}}, c=\frac{1}{\left|h_{m}\right|^{2}}, b=\eta P \lambda\left(\mathbf{H}_{B M} \mathbf{H}_{B M}^{H}\right) .
$$

The derivative of $f(\alpha)$ w.r.t. $\alpha$ is

$$
\frac{d f(\alpha)}{d \alpha}=-\log _{2}(g(\alpha))-\frac{b \beta g(\alpha)^{-1}}{(1-\alpha) \log (2)}\left(c+\frac{\alpha b}{1-\alpha}\right)^{-2}
$$

where $g(\alpha)=1+\frac{\beta}{c+\frac{\alpha b}{1-\alpha}} \geq 0, \forall \alpha \in[0,1]$. It is clear from (12) that $\frac{d f(\alpha)}{d \alpha}<0$ for all $\alpha$, i.e., $f(\alpha)$ is a monotonically decreasing function of $\alpha$. This means that maximum of the objective function is achieved when $\alpha$ is minimum provided that the equality constraint is fulfilled. However, as $\alpha \rightarrow 0$, $\Gamma_{B} \rightarrow \infty$, i.e., the infeasibility of the SDR optimization problem (9) increases. Consequently, the optimum $\alpha$ is the minimum $\alpha$ for which (9) is feasible. The output $\mathbf{V}_{B}$ of such feasible SDR provides the optimum $\mathbf{w}_{B}$. In a nutshell, the proposed optimum solution can be summarized as follows:

- 1) Define a fine grid of $\alpha$ in steps of $\partial \alpha$.

- 2) Solve (9) with the smallest $\alpha$.

- 3) If feasible, stop and output $\alpha$ and $\mathbf{V}_{B}$.

- 4) If not, repeat step (2) with the increment of $\partial \alpha$.

\section{B. Suboptimal Method}

As a suboptimal method of optimizing $\mathbf{w}_{B}$ and $\alpha$, we consider ZF approach. This requires that

$$
\mathbf{w}_{B}^{H} \mathbf{H}_{B}^{H} \mathbf{h}_{M}=0 .
$$

1) Optimization of $\mathbf{w}_{B}$ : Substituting (13) into (6), the resulting optimization problem is expressed as

$$
\begin{aligned}
\max _{\left\|\mathbf{w}_{B}\right\|^{2} \leq P, 0 \leq \alpha \leq 1} & (1-\alpha) \log \left(1+\frac{\left|\mathbf{h}_{B}^{H} \mathbf{w}_{B}\right|^{2}}{1+p_{m}\left|h_{m}\right|^{2}}\right) \\
\text { s.t. } & (1-\alpha) \log \left(1+p_{m}\left\|\mathbf{h}_{M}\right\|^{2}\right)=R_{B} \\
& p_{m}=\frac{\alpha \eta P \lambda\left(\mathbf{H}_{B M} \mathbf{H}_{B M}^{H}\right)}{1-\alpha} \\
& \mathbf{w}_{B}^{H} \mathbf{H}_{B}^{H} \mathbf{h}_{M}=0 .
\end{aligned}
$$

For a given $\alpha$, the optimization of $\mathbf{w}_{B}$ becomes

$$
\begin{array}{cl}
\max _{\mathbf{w}_{B}} & \left|\mathbf{h}_{B}^{H} \mathbf{w}_{B}\right|^{2} \\
\text { s.t. } & \left\|\mathbf{w}_{B}\right\|^{2} \leq P \\
& \mathbf{w}_{B}^{H} \mathbf{H}_{B}^{H} \mathbf{h}_{M}=0 .
\end{array}
$$

Using a standard Lagrangian multiplier method and skipping the corresponding details, the closed-form solution of $\mathbf{w}_{B}$ is expressed as

$$
\mathbf{w}_{B}=\sqrt{P} \frac{\mathbf{B} \mathbf{h}_{B}}{\left\|\mathbf{B} \mathbf{h}_{B}\right\|}, \mathbf{B}=\mathbf{I}-\frac{\mathbf{H}_{B}^{H} \mathbf{h}_{M} \mathbf{h}_{M}^{H} \mathbf{H}_{B}}{\left\|\mathbf{H}_{B}^{H} \mathbf{h}_{M}\right\|^{2}}
$$

which is independent of $\alpha$. Consequently, the corresponding objective function is

$$
\left|\mathbf{h}_{B}^{H} \mathbf{w}_{B}\right|^{2}=P \frac{\left|\mathbf{h}_{B}^{H} \mathbf{B} \mathbf{h}_{B}\right|^{2}}{\left\|\mathbf{B} \mathbf{h}_{B}\right\|^{2}}=P\left\|\mathbf{B} \mathbf{h}_{B}\right\|^{2} .
$$

2) Optimization of $\alpha$ : Denote the suboptimal beamformer solution of (16) by $\mathbf{w}_{B}^{*}$. The remaining optimization problem w.r.t. $\alpha$ is expressed as

$$
\begin{array}{cc}
\max _{0 \leq \alpha \leq 1} & f(\alpha) \triangleq(1-\alpha) \log _{2}\left(1+\frac{\beta}{c+\frac{\alpha b}{1-\alpha}}\right) \\
\text { s.t. } & (1-\alpha) \log _{2}\left(1+\frac{\alpha}{1-\alpha} b \gamma\right)=R_{B} .
\end{array}
$$

where $\gamma=\left\|\mathbf{h}_{M}\right\|^{2}$. Note that the optimum $\alpha$ would be zero if there were no equality constraint (or the constraint with $R_{B}=0$ ). In the presence of equality constraint with $R_{B}>0$, it is clear that the optimum $\alpha$ is the smallest $\alpha$ that satisfies the equality constraint. The following proposition derives the optimum $\alpha$.

Proposition 1. When equality constraint is feasible (i.e., $R_{B} \leq R_{B}^{\max }$ ), the optimum $\alpha$ is given by

$$
\alpha^{\mathrm{opt}}=\frac{-\frac{1}{R_{B} \log (2)} W\left(-\frac{R_{B} \log (2)}{b \gamma} \mathrm{e}^{R_{B} \log (2)\left(1-\frac{1}{b \gamma}\right)}\right)-\frac{1}{b \gamma}}{1-\frac{1}{R_{B} \log (2)} W\left(-\frac{R_{B} \log (2)}{b \gamma} \mathrm{e}^{R_{B} \log (2)\left(1-\frac{1}{b \gamma}\right)}\right)-\frac{1}{b \gamma}}
$$

where $W(y)$ is the Lambert function, i.e., $y=x \mathrm{e}^{x} \Longrightarrow x=$ $W(y)$.

Proof. The proof is given in Appendix VI-B. 


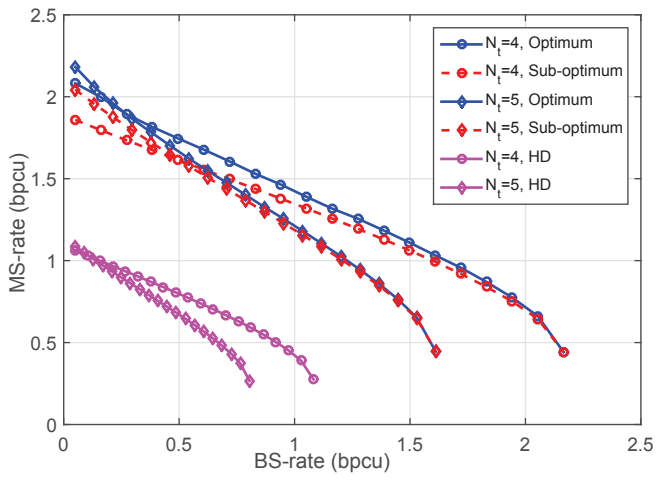

Fig. 1. Comparison of rate regions with $P=0 \mathrm{~dB}$, and $N_{t}=4,5$

\section{Simulation Results}

In all simulation results, we take $N=6$ and change the value of $N_{t}$. The channel coefficients for all channels are taken as zero-mean independent and identically distributed complex Gaussian random variables with unit variance. All results correspond to averaging of 100 independent channel realizations. The BS rate is varied from 0 to $R_{B}^{\max }$, where $R_{B}^{\max }$ is computed as in Appendix VI-A. The noise powers at both the BS and MS are set to unity.

Fig. 1 shows the rate regions obtained with the optimum and suboptimum methods for $N_{t}=4$ and 5 , when $P=0$ $\mathrm{dB}$, whereas the corresponding regions for $P=10 \mathrm{~dB}$ are shown in Fig. 2. As a benchmark, the achieved BS-MS rate regions are also shown for the HD mode. It can be observed from Figs. 1 and 2 that the maximum value of the MS rate is obtained when $R_{B}$ is minimum, whereas the minimum value is obtained when $R_{B}$ takes maximum value. Moreover, as expected both the BS and MS rates increase when $P$ increases from $0 \mathrm{~dB}$ to $10 \mathrm{~dB}$. Both figures show that the optimum method performs better than the suboptimum approach. However, the advantage of the optimum method over the suboptimum method diminishes when $P$ increases from $0 \mathrm{~dB}$ to $10 \mathrm{~dB}$. Moreover, when $N_{t}$ increases, the obtained maximum MS rate increases, whereas the obtained maximum BS rate decreases. This can be explained from the fact that increasing $N_{t}$ improves the transmit beamforming at the BS, which in turn is attributed for an increase in the MS rate. However, increase in $N_{t}$ decreases $N_{r}=N-N_{t}$ for a given $N_{t}$. This means that the LI rejection capability of the BS decreases which leads to a drop in the supported BS rate. All results also show that the FD operation almost doubles the rate of the HD mode.

The rate regions of the optimum and suboptimum methods with different values of $N_{t}=2$ and 3 are shown in Fig. 3 and Fig. 4 for $P=0 \mathrm{~dB}$ and $P=10 \mathrm{~dB}$, respectively. From these figures, similar observations can be made as in Figs. 1 and Fig. 2. However, in contrast to the latter figures, Figs. 3 and 4 show a significant performance gains for the optimum method compared to the suboptimum method. More specifically, the advantage of the optimum method is more pronounced for smaller values of $N_{t}$ and $P$.

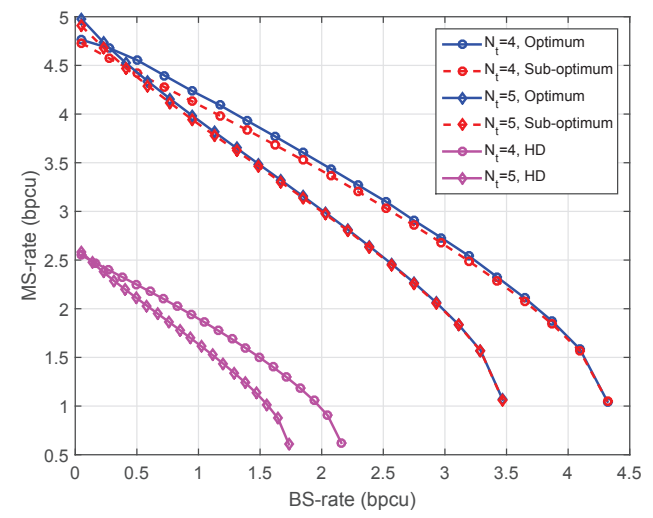

Fig. 2. Comparison of rate regions with $P=10 \mathrm{~dB}$, and $N_{t}=4,5$.

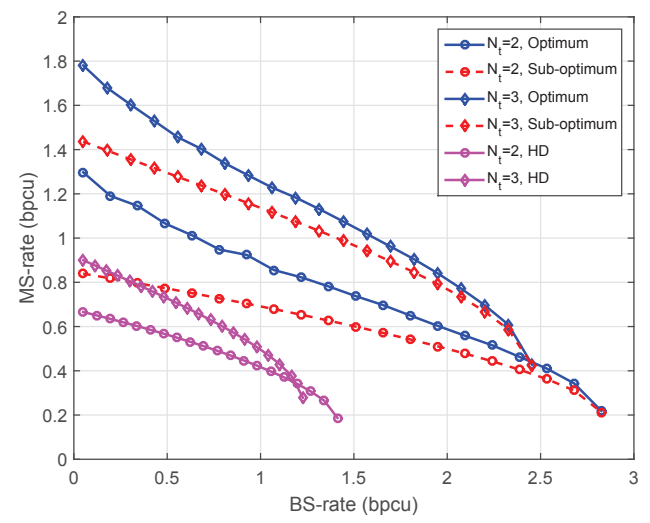

Fig. 3. Comparison of rate regions with $P=0 \mathrm{~dB}$, and $N_{t}=2,3$.

\section{CONCLUSiOnS}

In this paper, computationally efficient optimum and suboptimum methods were proposed to enlarge the boundary of the BS-MS rate region for a bi-directional FD communication system equipped with an $N$-antenna BS and a wirelesspowered MS equipped with two antennas. Simulation results demonstrate that significant performance gains are achievable when the BS-beamformer and the time-splitting parameter, which splits the available time between energy harvesting

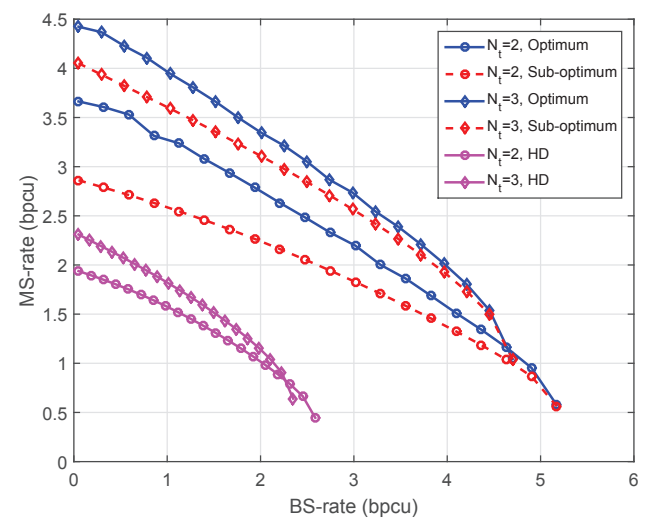

Fig. 4. Comparison of rate regions $P=10 \mathrm{~dB}$, and $N_{t}=2,3$. 
and FD communication modes, are jointly optimized. The advantages of multi-antenna transmission for LI suppression were also demonstrated.

\section{APPENDIX}

\section{A. Derivation of maximum $B S$ rate $R_{B}^{\max }$}

It is obvious that

$$
\left\|\mathbf{h}_{M}\right\|^{2}-\frac{\left|\mathbf{h}_{M}^{H} \mathbf{H}_{B} \mathbf{w}_{B}\right|^{2}}{1+\left\|\mathbf{H}_{B} \mathbf{w}_{B}\right\|^{2}} \leq\left\|\mathbf{h}_{M}\right\|^{2}
$$

where the equality is achieved with the $\mathrm{ZF}$ constraint $\mathbf{h}_{M}^{H} \mathbf{H}_{B} \mathbf{w}_{B}=0$. The maximum BS rate is then obtained as

$$
R_{B}^{\max }=\max _{0<\alpha<1}(1-\alpha) \log _{2}\left(1+\frac{\alpha}{1-\alpha} b\left\|\mathbf{h}_{M}\right\|^{2}\right)
$$

where $b=\eta P \lambda\left(\mathbf{H}_{B M} \mathbf{H}_{B M}^{H}\right)$. Denote $\tilde{b}=b\left\|\mathbf{h}_{M}\right\|^{2}$. Equating the first order derivative of $R_{B}^{\max }$ w.r.t. $\alpha$, we obtain

$$
\frac{\partial R_{B}^{\max }}{\partial \alpha}=0 \Longrightarrow \log \left(1+\frac{\alpha}{1-\alpha} \tilde{b}\right)=\frac{\tilde{b}}{1+\frac{\alpha}{1-\alpha} \tilde{b}} \frac{1}{1-\alpha}
$$

which can be written in the form

$$
z \log (z)=z+\tilde{b}-1, \text { where } z=1+\frac{\alpha}{1-\alpha} \tilde{b} .
$$

After straightforward manipulation, we obtain

$$
\frac{z}{\mathrm{e}} \log \left(\frac{z}{\mathrm{e}}\right)=\frac{\tilde{b}-1}{\mathrm{e}} \Longrightarrow \log \left(\frac{z}{\mathrm{e}}\right) \mathrm{e}^{\log \left(\frac{z}{\mathrm{e}}\right)}=\frac{\tilde{b}-1}{\mathrm{e}} .
$$

According to the definition of Lambert-W function, the solution of the equation $y=x e^{x}$ for a given $y$ is expressed as $x=W(y)$, where $W(\cdot)$ is the Lambert-W function. Thus, (24) is given by

$$
z=\mathrm{e}^{W\left(\frac{\tilde{b}-1}{\mathrm{e}}\right)+1} .
$$

Substituting $z$ into (25), the optimum $\alpha$ is

$$
\alpha^{\mathrm{Opt}}=\frac{\mathrm{e}^{W\left(\frac{\tilde{b}-1}{\mathrm{e}}\right)+1}-1}{\tilde{b}+\mathrm{e}^{W\left(\frac{\tilde{b}-1}{\mathrm{e}}\right)+1}-1} .
$$

Therefore, $R_{B}^{\max }$ is given by

$$
R_{B}^{\max }=\left(1-\alpha^{\mathrm{Opt}}\right) \log _{2}\left(1+\frac{\alpha^{\mathrm{Opt}}}{1-\alpha^{\mathrm{Opt}}} b\left\|\mathbf{h}_{M}\right\|^{2}\right) .
$$

\section{B. Proof of Proposition 1}

Proof. The equality constraint for the BS rate is expressed as

$$
\log \left(1+\frac{\alpha}{1-\alpha} b \gamma\right)=R_{B} \log (2)\left(\frac{\alpha}{1-\alpha}+1\right) .
$$

Define $y \triangleq 1+\frac{\alpha}{1-\alpha} b \gamma$. Then (28) can be expressed in terms of $y$ as

$$
y=\mathrm{e}^{\frac{R_{B} \log (2)}{b \gamma} y} \mathrm{e}^{R_{B} \log (2)\left(1-\frac{1}{b \gamma}\right)}
$$

which after simple manipulation is expressed as

$$
\begin{aligned}
\left(-\frac{R_{B} \log (2)}{b \gamma} y\right) \mathrm{e}^{-\frac{R_{B} \log (2)}{b \gamma} y}= & \left(-\frac{R_{B} \log (2)}{b \gamma}\right) \\
& \times \mathrm{e}^{R_{B} \log (2)\left(1-\frac{1}{b \gamma}\right)} .
\end{aligned}
$$

Using the Lambert-W function $W(y)$ (i.e., $y=x \mathrm{e}^{x} \rightarrow x=$ $W(y)), y$ in (30) is expressed as

$$
y=\frac{-b \gamma}{R_{B} \log (2)} W\left(-\frac{R_{B} \log (2)}{b \gamma} \mathrm{e}^{R_{B} \log (2)\left(1-\frac{1}{b \gamma}\right)}\right) .
$$

Note that $\frac{R_{B} \log (2)}{b \gamma} \mathrm{e}^{R_{B} \log (2)\left(1-\frac{1}{b \gamma}\right)} \leq \frac{1}{\mathrm{e}}$ is required to have a real value of $y$. If not, the equality constraint is not feasible for given $b, \gamma$, and $R_{B}$ where $R_{B} \leq R_{B}^{\max }$. Substituting $y$ in (31), we obtain

$\frac{\alpha}{1-\alpha}=\frac{-1}{R_{B} \log (2)} W\left(-\frac{R_{B} \log (2)}{b \gamma} \mathrm{e}^{R_{B} \log (2)\left(1-\frac{1}{b \gamma}\right)}\right)-\frac{1}{b \gamma}$

which yields the optimum $\alpha^{\text {Opt }}$ given in (19).

\section{REFERENCES}

[1] A. Sabharwal et al., "In-band full-duplex wireless: Challenges and opportunities," IEEE J. Sel. Areas Commun., vol. 32, pp. 1637-1652, Sep. 2014.

[2] T. Riihonen, S. Werner, and R. Wichman, "Mitigation of loopback selfinterference in full-duplex MIMO relays," IEEE Trans. Signal Process., vol. 59, pp. 5983-5993, Dec. 2011.

[3] B. P. Day, A. R. Margetts, D. W. Bliss, and P. Schniter, "Full-duplex bidirectional MIMO: Achievable rates under limited dynamic range," IEEE Trans. Signal Process., vol. 60, pp. 3702-3713, Jul. 2012.

[4] M. Duarte, "Full-duplex wireless: Design, implementation and characterization," Ph.D. Dissertation, Dept. Elect. and Computer Eng., Rice University, Houston, TX, 2012.

[5] H. A. Suraweera, I. Krikidis, G. Zheng, C. Yuen and P. J. Smith, "Lowcomplexity end-to-end performance optimization in MIMO full-duplex relay systems," IEEE Trans. Wireless Commun., vol. 13, pp. 913-927, Feb. 2014.

[6] H. Q. Ngo, H. A. Suraweera, M. Matthaiou and E. G. Larsson, "Multipair full-duplex relaying with massive arrays and linear processing," IEEE J. Sel. Areas Commun., vol. 32, pp. 1721-1737, Sep. 2014.

[7] R. Zhang and C. Ho, "MIMO broadcasting for simultaneous wireless information and power transfer," IEEE Trans. Wireless Commun., vol. 12, pp. 1989-2001, May 2013.

[8] Z. Ding, C. Zhong, D. W. K. Ng, M. Peng, H. A. Suraweera, R. Schober and H. V. Poor, "Application of smart antenna technologies in simultaneous wireless information and power transfer," IEEE Commun. Mag., vol. 53, pp. 86-93, Apr. 2015.

[9] K. Huang and X. Zhou, "Cutting last wires for mobile communication by microwave power transfer," IEEE Commun. Mag., vol. 53, pp. 86-93, June 2015.

[10] D. Senaratne and C. Tellambura, "Beamforming for space division duplexing," in Proc. IEEE ICC 2011, Kyoto, Japan, June 2011, pp. 1-5.

[11] H. Ju, X. Shang, H. V. Poor and D. Hong, "Bi-directional use of spatial resources and effects of spatial correlation," IEEE Trans. Wireless Commun., vol. 10, pp. 3368-3379, Oct. 2011.

[12] A. C. Cirik, Y. Rong and Y. Hua, "Achievable rates of full-duplex MIMO radios in fast fading channels with imperfect channel estimation," IEEE Trans. Signal Process., vol. 62, pp. 3874-3886, Aug. 2014.

[13] H. Ju and R. Zhang, "Optimal resource allocation in full-duplex wireless powered communication network," IEEE Trans. Commun., vol. 62, pp. 3528-3540, Oct. 2014.

[14] K. Yamazaki, Y. Sugiyama, Y. Kawaharay, S. Saruwatari and T. Watanabe, "Preliminary evaluation of simultaneous data and power transmission in the same frequency channel," in Proc. IEEE WCNC 2015, New Orleans, LA, Mar. 2015, pp. 1-6.

[15] Z. Hu, C. Yuan, F. Zhu, and F. Gao, "Weighted sum transmit power minimization for full-duplex system with SWIPT and self-energy recycling," [Online]. Available: http://arxiv.org/abs/1512.08311

[16] B. K. Chalise, W.-K. Ma, Y. D. Zhang, H. A. Suraweera, and M. G. Amin, "Optimum performance boundaries of OSTBC based AFMIMO relay system with energy harvesting receiver," IEEE Trans. Signal Process., vol. 61, no. 17. pp. 4199-4213, Sept. 2013. 